\title{
NEUTRAL SUBSPACES OF PAIRS OF SYMMETRIC/SKEWSYMMETRIC REAL MATRICES*
}

\author{
LEIBA RODMAN ${ }^{\dagger}$ AND PETER ŠEMRL ${ }^{\ddagger}$
}

\begin{abstract}
Let $A$ and $B$ be $n \times n$ real matrices with $A$ symmetric and $B$ skewsymmetric. Obviously, every simultaneously neutral subspace for the pair $(A, B)$ is neutral for each Hermitian matrix $X$ of the form $X=\mu A+i \lambda B$, where $\mu$ and $\lambda$ are arbitrary real numbers. It is well-known that the dimension of each neutral subspace of $X$ is at most $\operatorname{In}_{+}(X)+\operatorname{In}_{0}(X)$, and similarly, the dimension of each neutral subspace of $X$ is at most $\operatorname{In}_{-}(X)+\operatorname{In}_{0}(X)$. These simple observations yield that the maximal possible dimension of an $(A, B)$-neutral subspace is no larger than

$$
\min \left\{\min \left\{\operatorname{In}_{+}(\mu A+i \lambda B)+\operatorname{In}_{0}(\mu A+i \lambda B), \operatorname{In}_{-}(\mu A+i \lambda B)+\operatorname{In}_{0}(\mu A+i \lambda B)\right\}\right\}
$$

where the outer minimum is taken over all pairs of real numbers $(\lambda, \mu)$. In this paper, it is proven that the maximal possible dimension of an $(A, B)$-neutral subspace actually coincides with the above expression.
\end{abstract}

Key words. Symmetric matrix, Skewsymmetric matrix, Hermitian matrix, Inertia, Neutral subspace.

AMS subject classifications. 15A21, 15A22, 15B57.

1. Introduction and main result. Let $F$ be the field of real numbers $R$, or the field of complex numbers C. Denote by $\mathrm{F}^{m \times n}$ the set of $m \times n$ matrices with entries in $\mathrm{F}$, and let $(x, y)$ be the standard inner product in $\mathrm{F}^{n}$ (short for $\mathrm{F}^{n \times 1}$ ).

Let $A, B \in \mathrm{R}^{n \times n}$, where $A$ is symmetric and $B$ is skewsymmetric. A subspace $\mathcal{M} \subseteq \mathrm{R}^{n}$ is called simultaneously neutral for $A$ and $B$, or $(A, B)$-neutral, if

$$
(A x, y)=0, \quad(B x, y)=0 \quad \text { for all } x, y \in \mathcal{M} .
$$

Simultaneously neutral subspaces for a pair of real symmetric/skewsymmetric matrices, as well as those for a pair of complex hermitian matrices, play a key role in the theory of algebraic Riccati equations (see e.g. [7] and references therein), and in symmetric factorizations of matrix polynomials and rational matrix functions with

*Received by the editors on July 18, 2010. Accepted for publication on September 6, 2011. Handling Editor: Bryan L. Shader.

${ }^{\dagger}$ Department of Mathematics, College of William and Mary, Williamsburg, VA 23187-8795, USA (lxrodm@math.wm.edu). Research supported in part by Faculty Research Assignment and Plumeri Faculty Excellence Award Award at the College of William and Mary.

${ }^{\ddagger}$ Faculty of Mathematics and Physics, University of Ljubljana, Jadranska 19, SI-1000 Ljubljana, Slovenia (peter.semrl@fmf.uni-lj.si). Research supported in part by a grant from the Ministry of Science of Slovenia. 
certain symmetries $[2,3,5,6,10]$; in the latter application, the $(A, B)$-neutral subspaces have the additional property that they are $B^{-1} A$-invariant (and $B$ is assumed to be invertible). In this paper, we focus on the following problem: Find the maximal possible dimension of $(A, B)$-neutral subspaces for symmetric/skewsymmetric pairs of real matrices. We will describe this dimension in terms of inertia of complex hermitian matrices; we denote by

$$
\operatorname{In}(A)=\left(\operatorname{In}_{+}(A), \operatorname{In}_{-}(A), \operatorname{In}_{0}(A)\right)
$$

the inertia of a hermitian matrix $A \in \mathrm{C}^{n \times n}$. Thus, $\operatorname{In}_{+}(A), \operatorname{In}_{-}(A)$, and $\operatorname{In}_{0}(A)$ stand for the number of positive, negative, and zero eigenvalues of $A$, respectively, counted with multiplicities.

The following observation will be useful:

Lemma 1.1. Let $A, B \in \mathrm{R}^{n \times n}, A=A^{T}, B=-B^{T}$. Then $A+i B$ and $A-i B$ are similar, and in particular

$$
\operatorname{In}(A+i B)=\operatorname{In}(A-i B) .
$$

Proof. Observe that $x+i y \in \mathrm{C}^{n}$, where $x, y \in \mathrm{R}^{n}$, is an eigenvector of $A+i B$ corresponding to the eigenvalue $t \in \mathrm{R}$ if and only if $y+i x$ is an eigenvector of $A-i B$ corresponding to the same eigenvalue $t$. Clearly, the set of vectors $x_{1}+i y_{1}, \ldots, x_{p}+$ $i y_{p}$ is linearly independent if and only if the set $y_{1}+i x_{1}, \ldots, y_{p}+i x_{p}$ is linearly independent. Hence, $A+i B$ and $A-i B$ have the same eigenvalues with the same multiplicities.

We now state our main result:

Theorem 1.2. Let $A$ be symmetric, $B$ skewsymmetric, $A, B \in \mathrm{R}^{n \times n}$. Then the maximal dimension of an $(A, B)$-neutral subspace $\mathcal{M} \subseteq \mathrm{R}^{n}$ coincides with

(1.2) $\min \left\{\min \left\{\operatorname{In}_{+}(\mu A+i \lambda B)+\operatorname{In}_{0}(\mu A+i \lambda B), \operatorname{In}_{-}(\mu A+i \lambda B)+\operatorname{In}_{0}(\mu A+i \lambda B)\right\}\right\}$,

where the outer minimum is taken over all pairs of real numbers $(\lambda, \mu)$.

Thus, the maximal dimension of an $(A, B)$-neutral subspace is described in terms of inertia of suitable combinations of $A$ and $B$. Analogues of Theorem 1.2 in the context of pairs of complex or quaternionic hermitian matrices $A$ and $B$, where $\mu A+$ $i \lambda B$ of Theorem 1.2 is replaced by $\mu A+\lambda B$, have been obtained in $[9,11]$. We mention in passing that an analogue of Theorem 1.2 for pairs of real symmetric matrices fails, see [11] for more details. 
REMARK 1.3.

(1) Note that the inner minimum in (1.2) is attained at some nonzero $\left(\lambda_{0}, \mu_{0}\right)$; indeed, for $\lambda=\mu=0,(1.2)$ takes value $n$. Since

$$
\operatorname{In}_{+}(t X)+\operatorname{In}_{0}(t X)=\operatorname{In}_{+}(X)+\operatorname{In}_{0}(X), \quad X \in C^{n \times n}, \quad X=X^{*}, \quad t>0,
$$

and

$$
\operatorname{In}_{+}(X)+\operatorname{In}_{0}(X)=\operatorname{In}_{-}(-X)+\operatorname{In}_{0}(-X), \quad X \in \mathrm{C}^{n \times n}, \quad X=X^{*},
$$

we have that (1.2) is equal to

$$
\min _{0 \leq \alpha<2 \pi}\left\{\operatorname{In}_{+}((\cos \alpha) A+i(\sin \alpha) B)+\operatorname{In}_{0}((\cos \alpha) A+i(\sin \alpha) B)\right\} .
$$

(2) Note that (1.2) is also equal to

$$
\begin{aligned}
& \min \left\{\min _{t \in \mathrm{R}}\left\{\operatorname{In}_{+}(A+i t B)+\operatorname{In}_{0}(A+i t B)\right\}\right. \\
& \left.\min _{t \in \mathrm{R}}\left\{\operatorname{In}_{+}(-A+i t B)+\operatorname{In}_{0}(-A+i t B)\right\}\right\}
\end{aligned}
$$

as well as to the formula analogous to (1.4) with the roles of $A$ and $B$ interchanged. To verify that, one needs to observe that by the continuity of the spectrum there exists a real $M>0$ such that

$$
\begin{aligned}
\operatorname{In}_{+}(i B)+\operatorname{In}_{0}(i B) & \geq \operatorname{In}_{+}\left(\frac{1}{t} A+i B\right)+\operatorname{In}_{0}\left(\frac{1}{t} A+i B\right) \\
& =\operatorname{In}_{+}(A+i t B)+\operatorname{In}_{0}(A+i t B)
\end{aligned}
$$

for all real numbers $t>M$.

(3) It follows from (1.1) that (1.3) is actually equal to

$$
\min _{0 \leq \alpha \leq \pi}\left\{\operatorname{In}_{+}((\cos \alpha) A+i(\sin \alpha) B)+\operatorname{In}_{0}((\cos \alpha) A+i(\sin \alpha) B)\right\}
$$

The rest of the paper is devoted to the proof of Theorem 1.2. Preliminary results, including the canonical form for pairs of real symmetric/skewsymmetric matrices, are stated and sometimes proved in Sections 2 - 4. The proof of Theorem 1.2 itself is given in Sections 5 and 6.

We fix some notation. By $e_{1}, \ldots, e_{n}$ we denote the elements of the standard basis of $\mathrm{F}^{n}$, and by $\operatorname{span}\left(x_{1}, \ldots, x_{p}\right)$ the linear span of vectors $x_{1}, \ldots, x_{p}$. The symbol $\# \mathcal{G}$ stands for the cardinality of the set $\mathcal{G}$. We denote by $I_{k}$ and $0_{k}$ the $k \times k$ identity and zero matrices, respectively. 
2. Preliminaries on inertia of Hermitian matrices. If $X \in \mathrm{C}^{n \times n}$ is Hermitian, a subspace $\mathcal{M} \subseteq \mathrm{C}^{n}$ is said to be $X$-neutral if $(X x, y)=0$ for all $x, y \in \mathcal{M}$, or equivalently $(X x, x)=0$ for all $x \in \mathcal{M}$.

Proposition 2.1. Let $X \in \mathrm{C}^{n \times n}$ be hermitian. Then an $X$-neutral subspace $\mathcal{M} \subseteq \mathrm{C}^{n}$ is maximal, in the sense that no subspace properly containing $\mathcal{M}$ is $X$ neutral, if and only if

$$
\operatorname{dim}(\mathcal{M})=\min \left\{\operatorname{In}_{+}(X)+\operatorname{In}_{0}(X), \operatorname{In}_{-}(X)+\operatorname{In}_{0}(X)\right\}
$$

Proposition 2.1 is standard; see for example [4, Section 2.3], where it is proved under the additional assumption that $X$ is invertible.

LEMma 2.2. Let $X$ be Hermitian matrix which is block partitioned as follows:

$$
X=\left[\begin{array}{ccc}
0_{k} & 0 & X_{1} \\
0 & X_{0} & X_{2} \\
X_{1}^{*} & X_{2}^{*} & X_{3}
\end{array}\right], \quad \text { or } \quad X=\left[\begin{array}{ccc}
X_{3} & X_{2} & X_{1} \\
X_{2}^{*} & X_{0} & 0 \\
X_{1}^{*} & 0 & 0_{k}
\end{array}\right]
$$

where the block $X_{1}$ is $k \times k$ and invertible. Then

$$
\operatorname{In}_{0}(X)=\operatorname{In}_{0}\left(X_{0}\right), \quad \operatorname{In}_{ \pm}(X)=k+\operatorname{In}_{ \pm}\left(X_{0}\right) .
$$

Proof. Say $X$ is given by the first formula in (2.1). Replacing $X$ with $S X S^{*}$, where

$$
S=\left[\begin{array}{ccc}
I_{k} & 0 & 0 \\
-X_{2} X_{1}^{-1} & I & 0 \\
-\frac{1}{2} X_{3} X_{1}^{-1} & 0 & I
\end{array}\right]
$$

we may assume $X_{2}=0, X_{3}=0$. It is easy to see that

$$
\operatorname{In}_{ \pm}\left[\begin{array}{cc}
0 & X_{1} \\
X_{1}^{*} & 0
\end{array}\right]=k .
$$

Now (2.2) is obvious.

3. Properties of $\Phi_{\alpha}(A, B)$. In this section, we let $A, B \in \mathrm{R}^{n \times n}$, where $A=A^{T}$, $B=-B^{T}$.

For convenience, denote

$$
\Phi_{\alpha}(A, B):=\operatorname{In}_{+}((\cos \alpha) A+i(\sin \alpha) B)+\operatorname{In}_{0}((\cos \alpha) A+i(\sin \alpha) B), \quad 0 \leq \alpha<2 \pi .
$$


We list some elementary properties of the quantity $\Phi_{\alpha}(A, B)$.

LEMMA 3.1. (a) If $Q$ is any finite subset of $[0,2 \pi)$, then

$$
\min _{0 \leq \alpha<2 \pi}\left(\Phi_{\alpha}(A, B)\right)=\min _{0 \leq \alpha<2 \pi, \alpha \notin Q}\left(\Phi_{\alpha}(A, B)\right) .
$$

(b) Assume

$$
A=\left[\begin{array}{cc}
A_{1} & 0 \\
0 & A_{2}
\end{array}\right], \quad B=\left[\begin{array}{cc}
B_{1} & 0 \\
0 & B_{2}
\end{array}\right]
$$

If

$$
\Phi_{\alpha}\left(A_{1}, B_{1}\right)=\Phi_{\alpha^{\prime}}\left(A_{1}, B_{1}\right)
$$

for all $\alpha, \alpha^{\prime} \in[0,2 \pi) \backslash Q$, where $Q$ is a finite (or empty) set, then

$$
\min _{0 \leq \alpha<2 \pi}\left(\Phi_{\alpha}(A, B)\right)=\min _{0 \leq \alpha<2 \pi}\left(\Phi_{\alpha}\left(A_{1}, B_{1}\right)\right)+\min _{0 \leq \alpha<2 \pi}\left(\Phi_{\alpha}\left(A_{2}, B_{2}\right)\right) .
$$

Note that (3.2) is generally not valid without additional hypotheses on $A_{j}$ and $B_{j}(\operatorname{such}$ as $(3.1))$.

Proof. Proof of (a). Let $\alpha_{0} \in[0,2 \pi)$ be such that

$$
\min _{0 \leq \alpha<2 \pi}\left(\Phi_{\alpha}(A, B)\right)=\Phi_{\alpha_{0}}(A, B)
$$

Continuity of eigenvalues of a Hermitian matrix $X$ (as functions of the entries of $X$; it is assumed that the eigenvalues are arranged in the nondecreasing order) implies that

$$
\begin{gathered}
\operatorname{In}_{+}\left(\left(\cos \alpha_{0}\right) A+i\left(\sin \alpha_{0}\right) B\right)+\operatorname{In}_{0}\left(\left(\cos \alpha_{0}\right) A+i\left(\sin \alpha_{0}\right) B\right) \geq \\
\operatorname{In}_{+}((\cos \beta) A+i(\sin \beta) B)+\operatorname{In}_{0}((\cos \beta) A+i(\sin \beta) B)
\end{gathered}
$$

for all values of $\beta \in[0,2 \pi)$ sufficiently close to $\alpha_{0}$. However, (3.3) implies that the strict inequality is impossible in (3.4). Thus,

$$
\operatorname{In}_{+}((\cos \beta) A+i(\sin \beta) B)+\operatorname{In}_{0}((\cos \beta) A+i(\sin \beta) B)=\min _{0 \leq \alpha<2 \pi}\left(\Phi_{\alpha}(A, B)\right)
$$

for all $\beta$ sufficiently close to $\alpha_{0}$. We see that the minimum $\min _{0 \leq \alpha<2 \pi}\left(\Phi_{\alpha}(A, B)\right)$ is attained on a set that contains a nondegenerate interval. The statement (a) is now clear. 
Proof of (b). We obviously have

$$
\Phi_{\alpha}(A, B)=\Phi_{\alpha}\left(A_{1}, B_{1}\right)+\Phi_{\alpha}\left(A_{2}, B_{2}\right), \quad \forall \alpha \in[0,2 \pi) .
$$

So (the first equality follows from part (a)):

$\min _{0 \leq \alpha<2 \pi} \Phi_{\alpha}(A, B)=\min _{0 \leq \alpha<2 \pi, \alpha \notin Q} \Phi_{\alpha}(A, B)=\min _{0 \leq \alpha<2 \pi, \alpha \notin Q}\left(\Phi_{\alpha}\left(A_{1}, B_{1}\right)+\Phi_{\alpha}\left(A_{2}, B_{2}\right)\right)$

which by (3.1) is equal to

$$
\Phi_{\alpha^{\prime}}\left(A_{1}, B_{1}\right)+\min _{0 \leq \alpha<2 \pi, \alpha \notin Q} \Phi_{\alpha}\left(A_{2}, B_{2}\right),
$$

where $\alpha^{\prime} \in[0,2 \pi) \backslash Q$ is fixed. By part (a) we have

$$
\begin{gathered}
\Phi_{\alpha^{\prime}}\left(A_{1}, B_{1}\right)=\min _{\alpha \in[0,2 \pi)} \Phi_{\alpha}\left(A_{1}, B_{1}\right), \\
\min _{0 \leq \alpha<2 \pi, \alpha \notin Q} \Phi_{\alpha}\left(A_{2}, B_{2}\right)=\min _{0 \leq \alpha<2 \pi} \Phi_{\alpha}\left(A_{2}, B_{2}\right),
\end{gathered}
$$

and we are done.

REMARK 3.2. The result of Lemma 3.1 (with essentially the same proof) remains valid if the interval $[0,2 \pi)$ is replaced by any nondegenerate subinterval, with or without one of both endpoints, of $[0,2 \pi)$.

LEMma 3.3. Assume that $A$ and $B$ have the following block form

$$
A=\left[\begin{array}{ccc}
0_{k} & 0 & A_{1} \\
0 & A_{0} & A_{2} \\
A_{1}^{T} & A_{2}^{T} & A_{3}
\end{array}\right], \quad B=\left[\begin{array}{ccc}
0_{k} & 0 & B_{1} \\
0 & B_{0} & B_{2} \\
-B_{1}^{T} & -B_{2}^{T} & B_{3}
\end{array}\right]
$$

where the blocks $A_{1}$ and $B_{1}$ are $k \times k$. Assume furthermore that $(\cos \alpha) A_{1}+i(\sin \alpha) B_{1}$ is invertible for all but finitely many values $\alpha \in[0,2 \pi)$. Then

$$
\min _{0 \leq \alpha<2 \pi} \Phi_{\alpha}(A, B)=k+\min _{0 \leq \alpha<2 \pi} \Phi_{\alpha}\left(A_{0}, B_{0}\right)
$$

Proof. Let

$$
Q=\left\{\alpha \in[0,2 \pi):(\cos \alpha) A_{1}+i(\sin \alpha) B_{1} \text { is not invertible }\right\}
$$

By Lemma 3.1(a) we may replace the interval $[0,2 \pi)$ with $[0,2 \pi) \backslash Q$ in (3.5). By Lemma 2.2, $\Phi_{\alpha}(A, B)=k+\Phi_{\alpha}\left(A_{0}, B_{0}\right)$ for $\alpha \in[0,2 \pi) \backslash Q$, and (3.5) follows. 
4. Canonical form. We present here the known canonical form of real symmetric/skewsymmetric matrix pencils

$$
A+\lambda B, \quad A, B \in \mathrm{R}^{n \times n}, \quad A=A^{T}, \quad-B=B^{T}
$$

under R-congruence:

$$
A+\lambda B \mapsto S^{T} A S+\lambda S^{T} B S, \quad S \in \mathrm{R}^{n \times n} \text { is invertible. }
$$

(See e.g. [8] and references there.) The following notation will be used:

$$
\Xi_{2}=\left[\begin{array}{cc}
0 & 1 \\
-1 & 0
\end{array}\right] \text {; }
$$

$F_{q}$ is the $q \times q$ real symmetric matrix with 1 's in positions $(1, q),(2, q-1), \ldots,(q, 1)$ and zeros elsewhere;

$$
G_{q}=\left[\begin{array}{cc}
F_{q-1} & 0_{(q-1) \times 1} \\
0_{1 \times(q-1)} & 0_{1}
\end{array}\right],
$$

a $q \times q$ real symmetric matrix, and we take $G_{1}=0$; we denote by $J_{2 m}(a \pm i b)$, where $a$ and $b$ are real and $b>0$, the $2 m \times 2 m$ almost upper triangular real Jordan block of size $2 m \times 2 m$ having eigenvalues $a \pm i b$.

It will be convenient to list the elementary blocks first:

$(\operatorname{sss} 0)$

a square size zero matrix.

(sss1)

$$
G_{2 \varepsilon+1}+\lambda\left[\begin{array}{ccc}
0 & 0 & F_{\varepsilon} \\
0 & 0_{1} & 0 \\
-F_{\varepsilon} & 0 & 0
\end{array}\right]
$$

$(\operatorname{sss} 2)$

$$
F_{k}+\lambda\left[\begin{array}{ccc}
0 & 0 & 0 \\
0 & 0 & F_{\frac{k-1}{2}} \\
0 & -F_{\frac{k-1}{2}} & 0
\end{array}\right], \quad k \text { odd }
$$

$(\operatorname{sss} 3)$

$$
F_{k}+\lambda\left[\begin{array}{cccc}
0_{1} & 0 & 0 & 0 \\
0 & 0 & 0 & F_{\frac{k-2}{2}} \\
0 & 0 & 0_{1} & 0 \\
0 & -F_{\frac{k-2}{2}} & 0 & 0
\end{array}\right], \quad k \text { even. }
$$


$(\operatorname{sss} 4)$

$$
G_{\ell}+\lambda\left[\begin{array}{cc}
0 & F_{\ell / 2} \\
-F_{\ell / 2} & 0
\end{array}\right], \quad \ell \text { even. }
$$

(sss5)

$$
\left[\begin{array}{cc}
0 & G_{\ell / 2} \\
G_{\ell / 2} & 0
\end{array}\right]+\lambda\left[\begin{array}{cc}
0 & F_{\ell / 2} \\
-F_{\ell / 2} & 0
\end{array}\right], \quad \ell \text { even and } \ell / 2 \text { odd. }
$$

(sss6)

$$
\left[\begin{array}{cc}
0 & \gamma F_{\ell / 2}+G_{\ell / 2} \\
\gamma F_{\ell / 2}+G_{\ell / 2} & 0
\end{array}\right]+\lambda\left[\begin{array}{cc}
0 & F_{\ell / 2} \\
-F_{\ell / 2} & 0
\end{array}\right], \quad \ell \text { even, } \gamma \in \mathrm{R} \backslash\{0\}
$$

(sss7)

$$
\begin{aligned}
& {\left[\begin{array}{cccccc}
0 & 0 & \cdots & 0 & 0 & \nu \Xi_{2}^{m+1} \\
0 & 0 & \cdots & 0 & -\nu \Xi_{2}^{m+1} & -I_{2} \\
0 & 0 & \cdots & \nu \Xi_{2}^{m+1} & -I_{2} & 0 \\
\vdots & \vdots & . \cdot & \vdots & \vdots & \vdots \\
(-1)^{m-1} \nu \Xi_{2}^{m+1} & -I_{2} & 0 & \cdots & 0 & 0
\end{array}\right]} \\
& +\lambda\left[\begin{array}{ccccc}
0 & 0 & \cdots & 0 & \Xi_{2}^{m} \\
0 & 0 & \cdots & -\Xi_{2}^{m} & 0 \\
\vdots & \vdots & . \cdot & \vdots & \vdots \\
0 & (-1)^{m-2} \Xi_{2}^{m} & \cdots & 0 & 0 \\
(-1)^{m-1} \Xi_{2}^{m} & 0 & \cdots & 0 & 0
\end{array}\right], \quad \nu>0 .
\end{aligned}
$$

The pencil in (sss7) is $2 m \times 2 m$, where $m$ is a positive integer. We denote the pencil in (sss7) by

$$
\Omega_{2 m}(\nu)+\lambda \widetilde{\Omega}_{2 m}
$$

Note that the matrices $\Omega_{2 m}(\nu)$ and $\widetilde{\Omega}_{2 m}$ are symmetric and skewsymmetric, respectively, for every $m$ (and every real $\nu$ ). $(\operatorname{sss} 8)$

$$
\left[\begin{array}{cc}
0 & J_{2 m}(a \pm i b)^{T} \\
J_{2 m}(a \pm i b) & 0
\end{array}\right]+\lambda\left[\begin{array}{cc}
0 & I_{2 m} \\
-I_{2 m} & 0
\end{array}\right]
$$

where $a, b>0$. The matrix pencil here is $4 m \times 4 m$. 
THEOREM 4.1. Let $A+\lambda B$ be a real symmetric/skewsymmetric matrix pencil. Then $A+\lambda B$ is $\mathrm{R}$-congruent to a real symmetric/skewsymmetric pencil of the form

$$
\begin{gathered}
\left(A_{0}+\lambda B_{0}\right) \oplus \bigoplus_{j=1}^{r} \delta_{j}\left(F_{k_{j}}+\lambda\left[\begin{array}{ccc}
0 & 0 & 0 \\
0 & 0 & F_{\frac{k_{j}-1}{2}} \\
0 & -\frac{F_{k_{j}-1}}{2} & 0
\end{array}\right]\right) \\
\oplus \bigoplus_{t=1}^{p} \eta_{t}\left(G_{\ell_{t}}+\lambda\left[\begin{array}{cc}
0 & F_{\ell_{t} / 2} \\
-F_{\ell_{t} / 2} & 0
\end{array}\right]\right) \oplus \bigoplus_{u=1}^{q} \zeta_{u}\left(\Omega_{2 m_{u}}\left(\nu_{u}\right)+\lambda \widetilde{\Omega}_{2 m_{u}}\right) .
\end{gathered}
$$

Here, $A_{0}+\lambda B_{0}$ is a direct sum of blocks of types ( $\left.\operatorname{sss} 0\right)$, ( $\left.\operatorname{sss} 1\right)$, (sss3), (sss5), (sss6), and (sss8) in which several blocks of the same type and of different and/or the same sizes may be present, and the $k_{j}$ 's are odd positive integers, the $\ell_{t}$ 's are even positive integers, the $\nu_{u}$ 's are positive real numbers, $\delta_{j}, \eta_{t}, \zeta_{u}$ are signs \pm 1 , and the $m_{u}$ 's are positive integers.

The blocks in (4.1) and (4.2) are uniquely determined by $A+\lambda B$ up to a permutation of blocks.

Theorem 4.1 is found in many sources; see, for example, [8] for a detailed proof.

5. Proof of Theorem 1.2: particular case. In this section, we prove the following particular case of Theorem 1.2:

Theorem 5.1. Let $A=A^{T} \in \mathrm{R}^{m \times m}, B=-B^{T} \in \mathrm{R}^{m \times m}$ be of the form

$$
A=\left(\oplus_{j=1}^{q} \kappa_{j}\left(-\nu_{j} I_{2}\right)\right) \oplus I_{t}, \quad B=\left(\oplus_{j=1}^{q} \kappa_{j} \Xi_{2}\right) \oplus 0_{t},
$$

where $t$ is a nonnegative integer, $\nu_{j}$ are positive numbers, $\kappa_{j}$ are signs \pm 1 , and if $\nu_{j_{1}}=\nu_{j_{2}}$ then $\kappa_{j_{1}}=\kappa_{j_{2}}$. Then there exists an $(A, B)$-neutral subspace of dimension $\min _{0 \leq \alpha<2 \pi} \Phi_{\alpha}(A, B)$.

We will need preliminary results.

\section{LEMMA 5.2. Let}

$$
A=\left(\oplus_{j=1}^{q} \kappa_{j}\left(-\nu_{j} I_{2}\right)\right) \oplus I_{t_{1}} \oplus-I_{t_{2}} \in \mathrm{R}^{m \times m}, \quad B=\left(\oplus_{j=1}^{q} \tau_{j} \Xi_{2}\right) \oplus 0_{t_{1}+t_{2}} \in \mathrm{R}^{m \times m},
$$

where $t_{1}, t_{2}$ are nonnegative integers, $\nu_{j}$ are positive numbers, $\kappa_{j}$ and $\tau_{j}$ are signs \pm 1 , and if $\nu_{j_{1}}=\nu_{j_{2}}$ then $\kappa_{j_{1}}=\kappa_{j_{2}}$. Let

$$
\rho_{+}(A, B):=\min _{v \in \mathrm{R}}\left\{\operatorname{In}_{+}(A+v i B)+\operatorname{In}_{0}(A+v i B)\right\} .
$$

Then there exists an $A$-nonnegative $B$-neutral subspace $\mathcal{M}$ of $\mathrm{R}^{m \times m}$ of dimension $\rho_{+}(A, B)$. 
Recall that a subspace $\mathcal{M}$ is called $A$-nonnegative if $\langle A x, x\rangle \geq 0$ for all $x \in \mathcal{M}$.

Before the proof of the lemma, it will be convenient to consider an example first.

EXAmple 5.3. Let

$$
A_{0}=\nu^{\prime} I_{2} \oplus-\nu^{\prime \prime} I_{2}, \quad B_{0}=\tau^{\prime} \Xi_{2} \oplus \tau^{\prime \prime} \Xi_{2},
$$

where $\nu^{\prime}>\nu^{\prime \prime}>0$ and $\tau^{\prime}, \tau^{\prime \prime}= \pm 1$. It is easy to see that $\rho_{+}\left(A_{0}, B_{0}\right)=2$. Then there exists an $A_{0}$-nonnegative $B_{0}$-neutral subspace of dimension two, for example,

$$
\operatorname{span}\left\{\left[\begin{array}{l}
1 \\
0 \\
1 \\
0
\end{array}\right], \quad\left[\begin{array}{c}
0 \\
1 \\
0 \\
\pm 1
\end{array}\right]\right\}
$$

where the sign \pm 1 is taken +1 if $\tau^{\prime} \neq \tau^{\prime \prime}$ and -1 if $\tau^{\prime}=\tau^{\prime \prime}$.

Proof of Lemma 5.2. Without loss of generality, we assume that the $\nu_{j}$ are arranged in the nondecreasing order:

$$
\nu_{1} \leq \nu_{2} \leq \cdots \leq \nu_{q}
$$

Let $\kappa=\kappa_{1}$, and separate the blocks in (5.1) according to the signs:

$$
\begin{gathered}
\kappa_{j}=\kappa \quad \text { for } j=1,2, \ldots, p_{1} ; \\
\kappa_{j}=-\kappa \text { for } j=p_{1}+1, p_{1}+2, \ldots, p_{2} ; \\
\kappa_{j}=\kappa \text { for } j=p_{2}+1, p_{2}+2, \ldots, p_{3}
\end{gathered}
$$

and so on, and finally

$$
\kappa_{j}= \pm \kappa \quad \text { for } \quad j=p_{s-1}+1, p_{s-1}+2, \ldots, p_{s} .
$$

Here $1 \leq p_{1}<p_{2}<\cdots<p_{s}=q$. By the hypotheses of Lemma 5.2, $\nu_{p_{\ell}}<\nu_{p_{\ell}+1}$ for $\ell=1,2, \ldots, s-1$.

In view of Lemma 1.1 and Remark 3.2, we have

$$
\rho_{+}(A, B)=\min _{v \in \Omega}\left\{\operatorname{In}_{+}(A+v i B)+\operatorname{In}_{0}(A+v i B)\right\}
$$

where

$$
\Omega:=\left\{v: v>0 \text { and } v \notin\left\{\nu_{1}, \ldots, \nu_{q}\right\}\right\}
$$


and since $A+v i B$ is invertible for $v \in \Omega$, we also have

$$
\rho_{+}(A, B)=\min _{v \in \Omega}\left\{\operatorname{In}_{+}(A+v i B)\right\} .
$$

Letting

$$
A^{\prime}=\oplus_{j=1}^{q} \kappa_{j}\left(-\nu_{j} I_{2}\right), \quad B=\oplus_{j=1}^{q} \tau_{j} \Xi_{2},
$$

we clearly obtain

$$
\rho_{+}\left(A^{\prime}, B^{\prime}\right)+t_{1}=\rho_{+}(A, B) .
$$

On the other hand, if $\mathcal{M}^{\prime}$ is an $A^{\prime}$-nonnegative $B^{\prime}$-neutral subspace of dimension $\rho_{+}\left(A^{\prime}, B^{\prime}\right)$, then

$$
\left[\begin{array}{c}
\mathcal{M} \\
\mathrm{R}^{t_{1}} \\
0_{t_{2}}
\end{array}\right]
$$

is an $A$-nonnegative $B$-neutral subspace of dimension $\rho_{+}\left(A^{\prime}, B^{\prime}\right)+t_{1}$. So, using induction on the size of matrices $A$ and $B$, we may (and do) assume that $t_{1}=t_{2}=0$.

Observe that for $\tau= \pm 1$ and $\nu>0$, we have

$$
\operatorname{In}_{+}\left(\tau\left(-\nu I_{2}\right) \pm i v \Xi_{2}\right)= \begin{cases}0 & \text { if } 0 \leq v<\nu \text { and } \tau=1 \\ 1 & \text { if } v>\nu \text { and } \tau= \pm 1 \\ 2 & \text { if } 0 \leq v<\nu \text { and } \tau=-1\end{cases}
$$

Thus, for $v \in \Omega$ we have

$$
\begin{aligned}
\operatorname{In}_{+}(A+i v B) & =2 \#\left\{j=1,2, \ldots, q: \nu_{j}>v \text { and } \kappa_{j}=-1\right\} \\
& +\#\left\{j=1,2, \ldots, q: \nu_{j}<v\right\} \\
& =q+\#\left\{j=1,2, \ldots, q: \nu_{j}>v \text { and } \kappa_{j}=-1\right\} \\
& -\#\left\{j=1,2, \ldots, q: \nu_{j}>v \text { and } \kappa_{j}=1\right\} .
\end{aligned}
$$

Therefore,

$$
\begin{aligned}
\rho_{+}(A, B) & =q+\min _{v \in \Omega}\left\{\#\left\{j=1,2, \ldots, q: \nu_{j}>v \text { and } \kappa_{j}=-1\right\}\right. \\
& \left.-\#\left\{j=1,2, \ldots, q: \nu_{j}>v \text { and } \kappa_{j}=1\right\}\right\} .
\end{aligned}
$$

In particular, $\rho_{+}(A, B) \leq q$. We now consider several cases.

Case (a): Assume $\rho_{+}(A, B)=q$. Then in view of (5.3), $\#\left\{j=1,2, \ldots, q: \nu_{j}>v\right.$ and $\left.\kappa_{j}=-1\right\} \geq \#\left\{j=1,2, \ldots, q: \nu_{j}>v\right.$ and $\left.\left.\kappa_{j}=1\right\}\right\}$ 
for all $v \in \Omega$. So, rearranging blocks in $A$ and $B$ (this amounts to a simultaneous row and column permutation in $A$ and $B$ ), we can bring $A$ and $B$ to the following form:

$$
\begin{aligned}
& A^{\prime \prime}=\oplus_{j=1}^{q^{\prime}}\left(\nu_{j, 1} I_{2} \oplus-\nu_{j, 2} I_{2}\right) \bigoplus \oplus_{j=1}^{q^{\prime \prime}} \mu_{j} I_{2}, \\
& B^{\prime \prime}=\oplus_{j=1}^{q^{\prime}}\left(\tau_{j, 1} \Xi_{2} \oplus \tau_{j, 2} \Xi_{2}\right) \bigoplus \oplus_{j=1}^{q^{\prime \prime}} \gamma_{j} \Xi_{2},
\end{aligned}
$$

where $\nu_{j, 1}>\nu_{j, 2}>0$ for $j=1,2, \ldots, q^{\prime} ; \mu_{j}>0$ for $j=1,2, \ldots, q^{\prime \prime} ; \tau_{j, 1}, \tau_{j, 2}$ and $\gamma_{j}$ are signs $\pm 1 ; 2 q^{\prime}+q^{\prime \prime}=q$. Clearly, every pair $\mu_{j} I_{2}, \gamma_{j} \Xi_{2}$ produces a one-dimensional $\mu_{j} I_{2}-$ nonnegative $\gamma_{j} \Xi_{2}$-neutral subspace, for example span $\left[\begin{array}{l}1 \\ 0\end{array}\right]$, and every pair $\nu_{j, 1} I_{2} \oplus$ $-\nu_{j, 2} I_{2}, \tau_{j, 1} \Xi_{2} \oplus \tau_{j, 2} \Xi_{2}$ produces a two-dimensional $\left(\nu_{j, 1} I_{2} \oplus-\nu_{j, 2} I_{2}\right)$-nonnegative $\left(\tau_{j, 1} \Xi_{2} \oplus \tau_{j, 2} \Xi_{2}\right)$-neutral subspace in view of Example 5.3. Putting all these subspaces together we obtain an $A$-nonnegative $B$-neutral subspace of the requisite dimension $q$.

Case (b): Assume $\rho_{+}(A, B)<q$ and $\kappa_{p_{s}}=1$. Let

$$
A^{\prime}=\oplus_{j=1}^{q-1} \kappa_{j}\left(-\nu_{j} I_{2}\right), \quad B=\oplus_{j=1}^{q-1} \tau_{j} \Xi_{2} .
$$

Using formula analogous to (5.3) for the pair $A^{\prime}, B^{\prime}$, we have

$$
\begin{aligned}
\rho_{+}\left(A^{\prime}, B^{\prime}\right) & =q-1+\min _{v \in \Omega}\left\{\#\left\{j=1,2, \ldots, q: \nu_{j}>v \text { and } \kappa_{j}=-1\right\}\right. \\
& \left.-\#\left\{j=1,2, \ldots, q-1: \nu_{j}>v \text { and } \kappa_{j}=1\right\}\right\}
\end{aligned}
$$

which is equal to

$$
\begin{aligned}
q-1+ & \min \left\{\operatorname { m i n } _ { v \in \Omega , v < \nu _ { q } } \left\{\#\left\{j=1,2, \ldots, q: \nu_{j}>v \text { and } \kappa_{j}=-1\right\}\right.\right. \\
- & \left.\#\left\{j=1,2, \ldots, q-1: \nu_{j}>v \text { and } \kappa_{j}=1\right\}\right\}, \\
& \min _{v \in \Omega, v>\nu_{q}}\left\{\#\left\{j=1,2, \ldots, q: \nu_{j}>v \text { and } \kappa_{j}=-1\right\}\right. \\
- & \left.\left.\#\left\{j=1,2, \ldots, q-1: \nu_{j}>v \text { and } \kappa_{j}=1\right\}\right\}\right\} \\
= & q-1+\min \left\{\operatorname { m i n } _ { v \in \Omega , v < \nu _ { q } } \left\{\#\left\{j=1,2, \ldots, q: \nu_{j}>v \text { and } \kappa_{j}=-1\right\}\right.\right. \\
- & \left.\left.\#\left\{j=1,2, \ldots, q: \nu_{j}>v \text { and } \kappa_{j}=1\right\}+1\right\}, 0\right\} \\
= & q+\min \left\{\operatorname { m i n } _ { v \in \Omega , v < \nu _ { q } } \left\{\#\left\{j=1,2, \ldots, q: \nu_{j}>v \text { and } \kappa_{j}=-1\right\}\right.\right. \\
- & \left.\left.\#\left\{j=1,2, \ldots, q: \nu_{j}>v \text { and } \kappa_{j}=1\right\}\right\},-1\right\} .
\end{aligned}
$$

In turn, this is equal to $\rho_{+}(A, B)$ in view of the formula (5.3) and our assumption $\rho_{+}(A, B)<q$. Using the induction hypothesis, we find $A^{\prime}$-nonnegative $B^{\prime}$-neutral subspace $\mathcal{M}^{\prime}$ of dimension $\rho_{+}(A, B)$. Then

$$
\left[\begin{array}{c}
\mathcal{M}^{\prime} \\
0
\end{array}\right] \subset \mathrm{R}^{m}
$$


is an $A$-nonnegative $B$-neutral subspace of dimension $\rho_{+}(A, B)$.

Case (c): Assume $\rho_{+}(A, B)<q$ and $\kappa_{p_{s}}=-1$.

Define the matrices $A_{j}^{\prime}, B_{j}^{\prime}, j=1,2, \ldots, q$, as follows: $A_{j}^{\prime}$ is obtained from $A$ by replacing the block $\kappa_{j}\left(-\nu_{j} I_{2}\right)$ with $\kappa_{j}\left(-\nu_{j}\right)$ (leaving all other blocks in $A$ intact), and $B_{j}^{\prime}$ is obtained from $B$ by replacing the block $\tau_{j} \Xi_{2}$ with zero (leaving all other blocks in $B$ intact). Thus, $A_{j}^{\prime}, B_{j}^{\prime} \in \mathrm{R}^{(m-1) \times(m-1)}$. Since $A_{j}^{\prime}$, resp. $B_{j}^{\prime}$, is obtained from $A$, resp. $B$, by removing the $2(j-1)+1$ th row and column, the interlacing inequalities for eigenvalues of principal submatrices of Hermitian matrices yield

$$
\operatorname{In}_{+}(A+v i B)-1 \leq \operatorname{In}_{+}\left(A_{j}^{\prime}+i v B_{j}^{\prime}\right) \leq \operatorname{In}_{+}(A+v i B), \quad v \in \Omega, \quad j=1,2, \ldots, q,
$$

and therefore

$$
\rho_{+}(A, B)-1 \leq \rho\left(A_{j}^{\prime}, B_{j}^{\prime}\right) \leq \rho_{+}(A, B), \quad j=1,2, \ldots, q .
$$

On the other hand, a computation using (5.2) shows that for $j_{0}=1,2, \ldots, q$, and for $v \in \Omega$ :

$$
\begin{aligned}
\operatorname{In}_{+}\left(A_{j_{0}}^{\prime}+i v B_{j_{0}}^{\prime}\right) & =\#\left\{j=1,2, \ldots, q: j \neq j_{0}, \quad \nu_{j}>v \text { and } \kappa_{j}=-1\right\} \\
& +q-1+\chi_{j_{0}}-\#\left\{j=1,2, \ldots, q: j \neq j_{0}, \quad \nu_{j}>v \text { and } \kappa_{j}=1\right\}
\end{aligned}
$$

where $\chi_{j_{0}}=1$ if $\kappa_{j_{0}}=-1$ and $\chi_{j_{0}}=0$ if $\kappa_{j_{0}}=1$. Thus,

$$
\begin{gathered}
\rho_{+}\left(A_{j_{0}}^{\prime}, B_{j_{0}}^{\prime}\right)=q \\
+\min _{v \in \Omega}\left\{-1+\chi_{j_{0}}+\#\left\{j=1,2, \ldots, q: j \neq j_{0}, \quad \nu_{j}>v \text { and } \kappa_{j}=-1\right\}\right. \\
\left.-\#\left\{j=1,2, \ldots, q: j \neq j_{0}, \quad \nu_{j}>v \text { and } \kappa_{j}=1\right\}\right\} .
\end{gathered}
$$

If there is $j_{0}$ such that

$$
w:=\rho_{+}\left(A_{j_{0}}^{\prime}, B_{j_{0}}^{\prime}\right)=\rho_{+}(A, B)
$$

then we can use induction on the size $m \times m$ of the matrices $A$ and $B$ to show that there exists a $w$-dimensional $A_{j_{0}}^{\prime}$-nonnegative $B_{j_{0}}^{\prime}$-neutral subspace $\mathcal{M}_{j_{0}}$. Let $x_{1}, \ldots, x_{w} \in \mathrm{R}^{m-1}$ be a basis for $\mathcal{M}_{j_{0}}$, and write

$$
x_{\gamma}=\left[\begin{array}{c}
x_{\gamma, 1} \\
x_{\gamma, 2} \\
\vdots \\
x_{\gamma, m-1}
\end{array}\right], \quad \gamma=1,2, \ldots, w
$$


Let

$$
\widehat{x}_{1}, \ldots, \widehat{x}_{w} \in \mathrm{R}^{m}
$$

be obtained from $x_{1}, \ldots, x_{w}$, respectively, by inserting a zero between $x_{\gamma, 2\left(j_{0}-1\right)}$ and $x_{\gamma, 2\left(j_{0}-1\right)+1}, \gamma=1,2, \ldots, w$. Then the subspace

$$
\widehat{\mathcal{M}}_{j_{0}}:=\operatorname{span}\left\{\widehat{x}_{1}, \ldots, \widehat{x}_{w}\right\}
$$

is $w$-dimensional and $A$-nonnegative and $B$-neutral.

It remains therefore to consider the situation when

$$
\rho_{+}\left(A_{j_{0}}^{\prime}, B_{j_{0}}^{\prime}\right)<\rho_{+}(A, B) \quad \forall j_{0}=1,2, \ldots, q,
$$

(in this case, necessarily

$$
\left.\rho_{+}\left(A_{j_{0}}^{\prime}, B_{j_{0}}^{\prime}\right)+1=\rho_{+}(A, B) \quad \forall j_{0}=1,2, \ldots, q\right),
$$

in other words,

$$
\begin{gathered}
\min _{v \in \Omega}\left\{\#\left\{j=1,2, \ldots, q: \nu_{j}>v \text { and } \kappa_{j}=-1\right\}\right. \\
\left.-\#\left\{j=1,2, \ldots, q: \nu_{j}>v \text { and } \kappa_{j}=1\right\}\right\} \\
=1+\min _{v \in \Omega}\left\{-1+\chi_{j_{0}}+\#\left\{j=1,2, \ldots, q: j \neq j_{0}, \quad \nu_{j}>v \text { and } \kappa_{j}=-1\right\}\right. \\
\left.-\#\left\{j=1,2, \ldots, q: j \neq j_{0}, \quad \nu_{j}>v \text { and } \kappa_{j}=1\right\}\right\}
\end{gathered}
$$

holds for $j_{0}=1,2, \ldots, q$. Thus, we assume that (5.4) holds. As we will see, this leads to a contradiction.

Consider the function

$$
\begin{aligned}
f(v) & =\#\left\{j=1,2, \ldots, q: \nu_{j}>v \text { and } \kappa_{j}=-1\right\} \\
& -\#\left\{j=1,2, \ldots, q: \nu_{j}>v \text { and } \kappa_{j}=1\right\}
\end{aligned}
$$

where $v \in \Omega$. We have

$$
\rho_{+}(A, B)=\min _{v \in \Omega} f(v)+q .
$$

Select points $\lambda_{0}, \ldots, \lambda_{s}$ so that

$$
0<\lambda_{0}<\nu_{1}, \quad \nu_{p_{1}}<\lambda_{1}<\nu_{p_{1}+1}, \ldots, \nu_{p_{s-1}}<\lambda_{s-1}<\nu_{p_{s-1}+1}, \quad \nu_{p_{s}}<\lambda_{s} .
$$

Clearly, at least one of the points $\lambda_{j}, j=0,1, \ldots, s$, is a point of (global) minimum for $f$. Since $f\left(\lambda_{s}\right)+q=q>\rho_{+}(A, B)$, the point $\lambda_{s}$ is not a point of minimum. Also, it follows from our assumption $\kappa_{q}=-1$ that

$$
f\left(\lambda_{s}\right)<f\left(\lambda_{s-1}\right), \quad f\left(\lambda_{s-1}\right)>f\left(\lambda_{s-2}\right), \quad f\left(\lambda_{s-2}\right)<f\left(\lambda_{s-3}\right),
$$


and so on. So, only the points $\lambda_{s-2}, \lambda_{s-4}, \lambda_{s-6}, \ldots$ can be points of (global) minimum of $f$.

Suppose $s$ is odd; then $\kappa_{1}=-1, \chi_{1}=1$, and $\lambda_{0}$ is not a point of minimum for $f$. The right hand side of (5.4) with $j_{0}=1$ takes the form

$$
\begin{aligned}
& 1+\min _{v \in \Omega}\left\{\#\left\{j=1,2, \ldots, q: j \neq 1, \quad \nu_{j}>v \text { and } \kappa_{j}=-1\right\}\right. \\
& \left.-\#\left\{j=1,2, \ldots, q: \quad \nu_{j}>v \text { and } \kappa_{j}=1\right\}\right\} .
\end{aligned}
$$

Clearly the minimum is achieved at one of the points $\lambda_{0}, \ldots, \lambda_{s}$. Thus,

$$
\begin{aligned}
1 & +\min _{v \in \Omega}\left\{\#\left\{j=1,2, \ldots, q: j \neq 1, \quad \nu_{j}>v \text { and } \kappa_{j}=-1\right\}\right. \\
& \left.-\#\left\{j=1,2, \ldots, q: \quad \nu_{j}>v \text { and } \kappa_{j}=1\right\}\right\} \\
& =1+\min \left\{f\left(\lambda_{0}\right)-1, f\left(\lambda_{1}\right), \ldots, f\left(\lambda_{s}\right)\right\} \\
& =1+\min \left\{f\left(\lambda_{0}\right), f\left(\lambda_{1}\right), \ldots, f\left(\lambda_{s}\right)\right\}
\end{aligned}
$$

(because $\lambda_{0}$ is not a point of minimum for $f$ ), which is one more than the left hand side of (5.4), a contradiction with (5.4).

Thus, suppose $s$ is even. Then $\kappa_{1}=1$. In this case, we select $j_{0}$ so that $\kappa_{j_{0}}=1$, $\chi_{j_{0}}=0$. The right hand side of (5.4) takes the form

$$
\begin{gathered}
\min _{v \in \Omega}\left\{\#\left\{j=1,2, \ldots, q: \quad \nu_{j}>v \text { and } \kappa_{j}=-1\right\}\right. \\
\left.-\#\left\{j=1,2, \ldots, q: j \neq j_{0}, \quad \nu_{j}>v \text { and } \kappa_{j}=1\right\}\right\} .
\end{gathered}
$$

Let $\lambda_{y}$ be the point of (global) minimum of $f$ having the largest index $y$; then we let $j_{0}=j_{p_{y}}+1$. (Note that we cannot have $y=s$ because $\lambda_{s}$ is not a point of minimum of $f$.) Again, the minimal value of

$$
\begin{gathered}
\#\left\{j=1,2, \ldots, q: \quad \nu_{j}>v \text { and } \kappa_{j}=-1\right\} \\
-\#\left\{j=1,2, \ldots, q: j \neq j_{0}, \quad \nu_{j}>v \text { and } \kappa_{j}=1\right\},
\end{gathered}
$$

where $v \in \Omega$, is achieved at one of the points $\lambda_{s-2}, \lambda_{s-4}, \ldots$. So, (5.5) becomes

$$
\begin{gathered}
\min _{z=s-2, s-4, \ldots}\left\{\#\left\{j=1,2, \ldots, q: \quad \nu_{j}>\lambda_{z} \text { and } \kappa_{j}=-1\right\}\right. \\
\left.-\#\left\{j=1,2, \ldots, q: j \neq j_{0}, \quad \nu_{j}>\lambda_{z} \text { and } \kappa_{j}=1\right\}\right\} .
\end{gathered}
$$

By the choice of $j_{0}=j_{p_{y}}+1$, we see that (5.6) is equal to

$$
\begin{gathered}
1+\min _{z=s-2, s-4, \ldots}\left\{\#\left\{j=1,2, \ldots, q: \quad \nu_{j}>\lambda_{z} \text { and } \kappa_{j}=-1\right\}\right. \\
\left.-\#\left\{j=1,2, \ldots, q: \quad \nu_{j}>\lambda_{z} \text { and } \kappa_{j}=1\right\}\right\}
\end{gathered}
$$


which is one more than the left hand side of (5.4), a contradiction again.

The following result proved in [1] will be also needed for the proof of Theorem 5.1 .

Proposition 5.4. Let $A, B \in \mathrm{R}^{n \times n}, A=A^{T}, B=-B^{T}$. Assume that there exists a d-dimensional subspace $\mathcal{M} \subseteq \mathrm{R}^{n}$ which is simultaneously $A$-nonnegative, i.e., $(A x, x) \geq 0$ for every $x \in \mathcal{M}$, and B-neutral, i.e., $(B x, y)=0$ for all $x, y \in \mathcal{M}$. Assume also that there exists a d-dimensional subspace $\mathcal{M}^{\prime} \subseteq \mathrm{R}^{n}$ which is simultaneously A-nonpositive and B-neutral. Then there exists a d-dimensional $(A, B)$-neutral subspace.

Proof of Theorem 5.1. By Lemma 5.2, there exists an $A$-nonnegative $B$-neutral subspace of dimension $\rho_{+}(A, B)$, and analogously there exists an $A$-nonpositive $B$ neutral subspace of dimension $\rho_{+}(-A, B)$. Since (cf. Remark $1.3(1)$ and $\left.(2)\right)$

$$
d:=\min _{0 \leq \alpha<2 \pi} \Phi_{\alpha}(A, B)=\min \left\{\rho_{+}(A, B), \rho_{+}(-A, B)\right\},
$$

it follows that there exist an $A$-nonnegative $B$-neutral subspace and an $A$-nonpositive $B$-neutral subspace of the same dimension $d$. Now Proposition 5.4 implies that there exists a $d$-dimensional $(A, B)$-neutral subspace.

6. Proof of Theorem 1.2: general case. Since by Proposition 2.1 an $(A, B)$ neutral subspace cannot have dimension greater than (1.2), we only have to prove existence of an $(A, B)$-neutral subspace $\mathcal{M}$ having dimension $\min _{0 \leq \alpha<2 \pi} \Phi_{\alpha}(A, B)$.

First, note that Lemma 3.1 leads to the following observation:

Proposition 6.1. Under the hypotheses of Lemma 3.1 part (b), if there is an $\left(A_{j}, B_{j}\right)$-neutral subspace $\mathcal{M}_{j}$ of dimension $\min _{0 \leq \alpha<2 \pi} \Phi_{\alpha}\left(A_{j}, B_{j}\right), j=1,2$, then there is an $(A, B)$-neutral subspace $\mathcal{M}$ of dimension $\min _{0 \leq \alpha<2 \pi} \Phi_{\alpha}(A, B)$.

Proof. Let

$$
\mathcal{M}=\left[\begin{array}{c}
\mathcal{M}_{1} \\
0
\end{array}\right]+\left[\begin{array}{c}
0 \\
\mathcal{M}_{2}
\end{array}\right],
$$

and take advantage of (3.2).

Without loss of generality we may (and do) assume that $A+\lambda B$ is in the canonical form as presented in Theorem 4.1.

Let $v_{0} \times v_{0}$ be the size of the zero block (if present) in $A_{0}+\lambda B_{0}$, let $v_{1} \times v_{1}$ be the total size of blocks of types $(\operatorname{sss} 3),(\operatorname{sss} 5),(\operatorname{sss} 6),(\operatorname{sss} 8)$ (if present) in $A_{0}+\lambda B_{0}$, and let

$$
\left(2 \varepsilon_{1}+1\right) \times\left(2 \varepsilon_{1}+1\right), \ldots,\left(2 \varepsilon_{s}+1\right) \times\left(2 \varepsilon_{s}+1\right)
$$


be the sizes of blocks of type (sss1) (if present) in $A_{0}+\lambda B_{0}$.

We shall calculate inertia of linear combinations of matrices in the blocks of types $(\operatorname{sss} 0)-(\operatorname{sss} 8)$, and in each case show a neutral subspace of the requisite dimension. The calculations are straightforward.

(1) If $A^{\prime}+\lambda B^{\prime}$ is the block $(\operatorname{sss} 0)$, then

$\operatorname{In}_{+}\left((\cos \alpha) A^{\prime}+i(\sin \alpha) B^{\prime}\right)=\operatorname{In}_{-}\left((\cos \alpha) A^{\prime}+i(\sin \alpha) B^{\prime}\right)=0, \quad \forall \alpha \in[0,2 \pi)$.

Clearly, there exists an $\left(A^{\prime}, B^{\prime}\right)$-neutral subspace of dimension $\min _{0 \leq \alpha<2 \pi} \Phi_{\alpha}\left(A^{\prime}, B^{\prime}\right)$.

(2) If $A^{\prime}+\lambda B^{\prime}$ is the block (sss1), then

$\operatorname{In}_{+}\left((\cos \alpha) A^{\prime}+i(\sin \alpha) B^{\prime}\right)=\operatorname{In}_{-}\left((\cos \alpha) A^{\prime}+i(\sin \alpha) B^{\prime}\right)=\epsilon, \quad \forall \alpha \in[0,2 \pi)$,

and $\operatorname{span}\left(e_{\epsilon+1}, \ldots, e_{2 \epsilon+1}\right)$ is an $\left(A^{\prime}, B^{\prime}\right)$-neutral subspace of dimension equal to $\min _{0 \leq \alpha<2 \pi} \Phi_{\alpha}\left(A^{\prime}, B^{\prime}\right)=\epsilon+1$.

(3) If $A^{\prime}+\lambda B^{\prime}$ is the block (sss3), then

$\operatorname{In}_{+}\left((\cos \alpha) A^{\prime}+i(\sin \alpha) B^{\prime}\right)=\operatorname{In}_{-}\left((\cos \alpha) A^{\prime}+i(\sin \alpha) B^{\prime}\right)= \begin{cases}k / 2 & \text { if } \cos \alpha \neq 0, \\ k / 2-1 & \text { if } \cos \alpha=0,\end{cases}$ and $\operatorname{span}\left(e_{1}, \ldots, e_{k / 2}\right)$ is an $\left(A^{\prime}, B^{\prime}\right)$-neutral subspace of dimension equal to $\min _{0 \leq \alpha<2 \pi} \Phi_{\alpha}\left(A^{\prime}, B^{\prime}\right)=k / 2$.

(4) If $A^{\prime}+\lambda B^{\prime}$ is the block (sss5), then

$\operatorname{In}_{+}\left((\cos \alpha) A^{\prime}+i(\sin \alpha) B^{\prime}\right)=\operatorname{In}_{-}\left((\cos \alpha) A^{\prime}+i(\sin \alpha) B^{\prime}\right)= \begin{cases}\ell / 2 & \text { if } \sin \alpha \neq 0, \\ \ell / 2-1 & \text { if } \sin \alpha=0,\end{cases}$ and $\operatorname{span}\left(e_{1}, \ldots, e_{\ell / 2}\right)$ is an $\left(A^{\prime}, B^{\prime}\right)$-neutral subspace of dimension equal to $\min _{0 \leq \alpha<2 \pi} \Phi_{\alpha}\left(A^{\prime}, B^{\prime}\right)=\ell / 2$.

(5) If $A^{\prime}+\lambda B^{\prime}$ is the block (sss6), then

$$
\operatorname{In}_{+}\left((\cos \alpha) A^{\prime}+i(\sin \alpha) B^{\prime}\right)=\operatorname{In}_{-}\left((\cos \alpha) A^{\prime}+i(\sin \alpha) B^{\prime}\right)=\ell / 2, \quad \forall \alpha \in[0,2 \pi),
$$

and $\operatorname{span}\left(e_{1}, \ldots, e_{\ell / 2}\right)$ is an $\left(A^{\prime}, B^{\prime}\right)$-neutral subspace of dimension equal to $\min _{0 \leq \alpha<2 \pi} \Phi_{\alpha}\left(A^{\prime}, B^{\prime}\right)=\ell / 2$.

(6) If $A^{\prime}+\lambda B^{\prime}$ is the block ( $\left.\operatorname{sss} 8\right)$, then

$$
\operatorname{In}_{+}\left((\cos \alpha) A^{\prime}+i(\sin \alpha) B^{\prime}\right)=\operatorname{In}_{-}\left((\cos \alpha) A^{\prime}+i(\sin \alpha) B^{\prime}\right)=2 m, \quad \forall \alpha \in[0,2 \pi),
$$

and $\operatorname{span}\left(e_{1}, \ldots, e_{2 m}\right)$ is an $\left(A^{\prime}, B^{\prime}\right)$-neutral subspace of dimension equal to $\min _{0 \leq \alpha<2 \pi} \Phi_{\alpha}\left(A^{\prime}, B^{\prime}\right)=2 m$. 
(7) If

$$
\begin{aligned}
A^{\prime}+\lambda B^{\prime} & =\left(F_{k}+\lambda\left[\begin{array}{ccc}
0 & 0 & 0 \\
0 & 0 & F_{\frac{k-1}{2}} \\
0 & -F_{\frac{k-1}{2}} & 0
\end{array}\right]\right) \\
& \oplus-\left(F_{k^{\prime}}+\lambda\left[\begin{array}{ccc}
0 & 0 & 0 \\
0 & 0 & F_{\frac{k^{\prime}-1}{2}} \\
0 & -F_{\frac{k^{\prime}-1}{2}} & 0
\end{array}\right]\right),
\end{aligned}
$$

where $k, k^{\prime}$ are odd, then

$$
\begin{aligned}
\operatorname{In}_{+}\left((\cos \alpha) A^{\prime}+i(\sin \alpha) B^{\prime}\right) & =\operatorname{In}_{-}\left((\cos \alpha) A^{\prime}+i(\sin \alpha) B^{\prime}\right)=\frac{k+k^{\prime}}{2}, \\
& \forall \alpha \in[0,2 \pi) \text { such that } \cos \alpha \neq 0
\end{aligned}
$$

Thus, $\operatorname{span}\left(e_{1}, \ldots, e_{(k-1) / 2}, e_{(k+1) / 2}+e_{k+\left(k^{\prime}+1\right) / 2}, e_{k+1}, \ldots, e_{k+\left(k^{\prime}-1\right) / 2}\right)$ is an $\left(A^{\prime}, B^{\prime}\right)$-neutral subspace of dimension $\min _{0 \leq \alpha<2 \pi} \Phi_{\alpha}\left(A^{\prime}, B^{\prime}\right)=\frac{k+k^{\prime}}{2}$.

(8) If $A^{\prime}+\lambda B^{\prime}$ is the block ( $\left.\operatorname{sss} 4\right)$, then

$$
\begin{aligned}
\operatorname{In}_{+}\left((\cos \alpha) A^{\prime}+i(\sin \alpha) B^{\prime}\right) & =\operatorname{In}_{-}\left((\cos \alpha) A^{\prime}+i(\sin \alpha) B^{\prime}\right)=\ell / 2, \\
& \forall \alpha \in[0,2 \pi) \text { such that } \sin \alpha \neq 0,
\end{aligned}
$$

and $\operatorname{span}\left(e_{\ell / 2+1}, \ldots, e_{\ell}\right)$ is an $\left(A^{\prime}, B^{\prime}\right)$-neutral subspace of dimension equal to $\min _{0 \leq \alpha<2 \pi} \Phi_{\alpha}\left(A^{\prime}, B^{\prime}\right)=\ell / 2$.

(9) If $A^{\prime}+\lambda B^{\prime}$ is the block (sss7) (of size $2 m \times 2 m$ ), with $m$ even, then

$$
\begin{array}{r}
\operatorname{In}_{+}\left((\cos \alpha) A^{\prime}+i(\sin \alpha) B^{\prime}\right) \\
=\operatorname{In}_{-}\left((\cos \alpha) A^{\prime}+i(\sin \alpha) B^{\prime}\right)=m, \\
\forall \alpha \in[0,2 \pi) \text { such that } \tan \alpha \neq \pm \nu,
\end{array}
$$

and $\operatorname{span}\left(e_{1}, \ldots, e_{m}\right)$ is an $\left(A^{\prime}, B^{\prime}\right)$-neutral subspace of dimension equal to $\min _{0 \leq \alpha<2 \pi} \Phi_{\alpha}\left(A^{\prime}, B^{\prime}\right)=m$.

(10) Assume

$$
A^{\prime}+\lambda B^{\prime}=\xi_{1}\left(\Omega_{2 m_{1}}(\nu)+\lambda \widetilde{\Omega}_{2 m_{1}}\right) \oplus \xi_{2}\left(\Omega_{2 m_{2}}(\nu)+\lambda \widetilde{\Omega}_{2 m_{2}}\right),
$$

where $\nu>0, m_{1}, m_{2}$ are odd, and

$$
\xi_{1}(-1)^{\frac{m_{1}-1}{2}}=-\xi_{2}(-1)^{\frac{m_{2}-1}{2}} .
$$

Then

$$
\operatorname{In}_{+}\left((\cos \alpha) A^{\prime}+i(\sin \alpha) B^{\prime}\right)=\operatorname{In}_{-}\left((\cos \alpha) A^{\prime}+i(\sin \alpha) B^{\prime}\right)=m_{1}+m_{2}
$$


for all $\alpha \in[0,2 \pi)$ except those values for which $\tan \alpha= \pm \nu$. Indeed, a calculation shows that the direct sum of the middle $2 \times 2$ block in

$$
\xi_{1}\left((\cos \alpha) \Omega_{2 m_{1}}(\nu)+i(\sin \alpha) \widetilde{\Omega}_{2 m_{2}}\right)
$$

and of the middle $2 \times 2$ block in

$$
\xi_{2}\left((\cos \alpha) \Omega_{2 m_{2}}(\nu)+i(\sin \alpha) \widetilde{\Omega}_{2 m_{2}}\right)
$$

is

$$
\xi_{1}\left((\cos \alpha) \nu \Xi_{2}^{m_{1}+1}+i(\sin \alpha) \Xi_{2}\right) \oplus \xi_{2}\left((\cos \alpha) \nu \Xi_{2}^{m_{2}+1}+i(\sin \alpha) \Xi_{2}\right)
$$

Now (6.2) follows easily from (6.3). Also, the $4 \times 4$ matrix (6.3) has the following 2-dimensional neutral subspace $\mathcal{M}_{0}$ independent of $\alpha$ (the hypothesis (6.1) is essential here):

$$
\mathcal{M}_{0}=\left\{\begin{array}{lll}
\operatorname{span}\left(e_{1}+e_{3}, e_{2}+e_{4}\right) & \text { if } m_{1}=4 k+3, & m_{2}=4 \ell+3, \\
\operatorname{span}\left(e_{1}+e_{3}, e_{2}+e_{4}\right) & \text { if } m_{1}=4 k+1, & m_{2}=4 \ell+1, \\
\operatorname{span}\left(e_{1}+e_{4}, e_{2}+e_{3}\right) & \text { if } m_{1}=4 k+3, & m_{2}=4 \ell+1, \\
\operatorname{span}\left(e_{1}+e_{4}, e_{2}+e_{3}\right) & \text { if } m_{1}=4 k+1, & m_{2}=4 \ell+3,
\end{array}\right.
$$

where $k$ and $\ell$ are nonnegative integers. Let

$\mathcal{M}=\operatorname{span}\left(e_{1}, \ldots, e_{m_{1}-1}, e_{2 m_{1}+1}, \ldots, e_{2 m_{1}+m_{2}-1}, e_{m_{1}}+e_{2 m_{1}+m_{2}}, e_{m_{1}+1}+e_{2 m_{1}+m_{2}+1}\right)$

if $\left(m_{1}-1\right) / 2$ and $\left(m_{2}-1\right) / 2$ have the same parity, and

$\mathcal{M}=\operatorname{span}\left(e_{1}, \ldots, e_{m_{1}-1}, e_{2 m_{1}+1}, \ldots, e_{2 m_{1}+m_{2}-1}, e_{m_{1}}+e_{2 m_{1}+m_{2}+1}, e_{m_{1}+1}+e_{2 m_{1}+m_{2}}\right)$

if $\left(m_{1}-1\right) / 2$ and $\left(m_{2}-1\right) / 2$ have different parity. It follows from (6.4) that $\mathcal{M}$ is an $\left(A^{\prime}, B^{\prime}\right)$-neutral subspace of dimension $\min _{0 \leq \alpha<2 \pi} \Phi_{\alpha}\left(A^{\prime}, B^{\prime}\right)=m_{1}+m_{2}$.

Repeatedly using Proposition 6.1, items (1) - (10) above, and Theorem 4.1, and replacing if necessary $A$ and $B$ by $-A$ and $-B$, respectively, we see that the proof of Theorem 1.2 is reduced to the consideration of the following case:

$$
\begin{aligned}
A+\lambda B & =\oplus_{j=1}^{q} \xi_{j}\left(\Omega_{2 m_{j}}\left(\nu_{j}\right)+\lambda \widetilde{\Omega}_{2 m_{j}}\right) \\
& \oplus \oplus_{i=1}^{s}\left(F_{k_{i}}+\lambda\left[\begin{array}{ccc}
0 & 0 & 0 \\
0 & 0 & F_{\frac{k_{i}-1}{2}} \\
0 & -F_{\frac{k_{i}-1}{2}} & 0
\end{array}\right]\right),
\end{aligned}
$$

where $m_{1}, \ldots, m_{q}$ are odd and $k_{1}, \ldots, k_{s}$ are odd, and $\xi_{j}$ are signs \pm 1 (the cases when $q=0$, i.e, the first part of (6.5) is missing, or $s=0$, i.e., the second part of $(6.5)$ 
is missing, are not excluded); also, if $\nu_{j_{1}}=\nu_{j_{2}}$ then the signs of the corresponding blocks in (6.5) are the same.

Applying a suitable simultaneous permutation of rows and columns to $A+\lambda B$ in (6.5), we obtain $A^{\prime}+\lambda B^{\prime}$ in the following block form:

$$
A^{\prime}+\lambda B^{\prime}=\left[\begin{array}{ccc}
0_{k} & 0 & A_{1}+\lambda B_{1} \\
0 & A_{0}+\lambda B_{0} & * \\
A_{1}^{T}-\lambda B_{1}^{T} & * & *
\end{array}\right],
$$

where

$$
k=\left(\sum_{j=1}^{q}\left(m_{j}-1\right)\right)+\left(\sum_{i=1}^{s} \frac{k_{i}-1}{2}\right)
$$

In (6.6), $A_{1}+\lambda B_{1}$ is a $k \times k$ block diagonal matrix pencil with the diagonal blocks of the forms

$$
\left[\begin{array}{cccc}
\cdots & 0 & 0 & \nu_{j} \Xi_{2}^{m_{j}+1} \\
\cdots & 0 & -\nu_{j} \Xi_{2}^{m_{j}+1} & -I_{2} \\
\cdots & \nu_{j} \Xi_{2}^{m_{j}+1} & -I_{2} & 0 \\
. \cdot & \vdots & \vdots & \vdots
\end{array}\right]+\lambda\left[\begin{array}{cccc}
\cdots & 0 & 0 & \Xi_{2}^{m_{j}} \\
\cdots & 0 & -\Xi_{2}^{m_{j}} & 0 \\
\cdots & \Xi_{2}^{m_{j}} & 0 & 0 \\
. \cdot & \vdots & \vdots & \vdots
\end{array}\right]
$$

where $j=1,2, \ldots, q$, and of the forms

$$
F_{\frac{k_{i}-1}{2}}+\lambda G_{\frac{k_{i}-1}{2}}^{\prime}, \quad i=1,2, \ldots, s
$$

where

$$
G_{m}^{\prime}=\left[\begin{array}{cc}
0_{1} & 0 \\
0 & F_{m-1}
\end{array}\right] \in \mathrm{R}^{m \times m}
$$

and

$$
\begin{aligned}
A_{0}+\lambda B_{0} & :=\left(\oplus_{j=1}^{q} \xi_{j}(-1)^{\frac{m_{j}-1}{2}}\left(\nu_{j} \Xi_{2}^{m_{j}+1}+\lambda \Xi_{2}^{m_{j}}\right)\right) \oplus I_{s} \\
& =\left(\oplus_{j=1}^{q} \xi_{j}\left(-\nu_{j} I_{2}+\lambda \Xi_{2}\right)\right) \oplus I_{s} .
\end{aligned}
$$

Note that $(\cos \alpha) A_{1}+i(\sin \alpha) B_{1}$ is invertible for all but finitely many values of $\alpha \in$ $[0,2 \pi)$. By Lemma 3.3, we have

$$
\min _{0 \leq \alpha<2 \pi} \Phi_{\alpha}\left(A^{\prime}, B^{\prime}\right)=k+\min _{0 \leq \alpha<2 \pi} \Phi_{\alpha}\left(A_{0}, B_{0}\right)
$$

On the other hand, by Theorem 5.1, there exists an $\left(A_{0}, B_{0}\right)$-neutral subspace $\mathcal{M}_{0}$ of dimension $\min _{0 \leq \alpha<2 \pi} \Phi_{\alpha}\left(A_{0}, B_{0}\right)$. Then clearly

$$
\mathcal{M}:=\left[\begin{array}{c}
\mathrm{R}^{k} \\
0 \\
0
\end{array}\right]+\left[\begin{array}{c}
0 \\
\mathcal{M}_{0} \\
0
\end{array}\right]
$$


is an $\left(A^{\prime}, B^{\prime}\right)$-neutral subpace of dimension $k+\min _{0 \leq \alpha<2 \pi} \Phi_{\alpha}\left(A_{0}, B_{0}\right)$. In view of (6.7), we have proved Theorem 1.2 for the pair $(A, B)$.

\section{REFERENCES}

[1] A. Ben-Artzi, D. Ž. Djokovič, and L. Rodman. Totally isotropic subspaces for pairs of Hermitian forms and applications to Riccati equations. Linear Algebra Appl., 159:121-128, 1991.

[2] I. Gohberg, P. Lancaster, and L. Rodman. Spectral analysis of selfadjoint matrix polynomials. Ann. of Math., 112:33-71, 1980.

[3] I. Gohberg, P. Lancaster, and L. Rodman. Matrices and Indefinite Scalar Products. OT8, Birkhäuser, Basel, 1983.

[4] I. Gohberg, P. Lancaster, and L. Rodman. Indefinite Linear Algebra and Applications. Birkhäuser, Boston, 2006.

[5] P. Lancaster and L. Rodman. Invariant neutral subspaces for symmetric and skew real pairs. Canad. J. Math., 46:602-618, 1994.

[6] P. Lancaster and L. Rodman. Minimal symmetric factorizations of symmetric real and complex rational matrix functions. Linear Algebra Appl., 220:249-282, 1995.

[7] P. Lancaster and L. Rodman. Algebraic Riccati Equations. Oxford University Press, New York, 1995.

[8] P. Lancaster and L. Rodman. Canonical forms for symmetric/skew-symmetric real matrix pairs under strict equivalence and congruence. Linear Algebra Appl., 406:1-76, 2005.

[9] C.-K. Li and N.-S. Sze. Canonical forms, higher rank numerical ranges, totally isotropic subspaces, and matrix equations. Proc. Amer. Math. Soc., 136:3013-3023, 2008.

[10] A.C.M. Ran. Minimal factorization of selfadjoint rational matrix functions. Integral Equations Operator Theory, 5:850-869, 1982.

[11] L. Rodman. Isotropic subspaces for pairs of Hermitian matrices. Electron. J. Linear Algebra, 17:508-517, 2008. 